\title{
Synergistic effects of tetrandrine combined with ionizing radiation on a murine colorectal carcinoma-bearing mouse model
}

\author{
WEI-CHAN LIN ${ }^{1,2 *}$, WEI-HSUN WANG ${ }^{3-5 *}$, YI-HSIEN LIN ${ }^{6}$, JYH-DER LEU $^{7}$, SHAN-YUN CHENG ${ }^{8}$, \\ YU-JEN CHEN $^{9}$ and JENG-JONG HWANG ${ }^{8}$
}

\begin{abstract}
${ }^{1}$ Department of Radiology, Cathay General Hospital, Taipei 106; ${ }^{2}$ School of Medicine, Fu-Jen Catholic University, New Taipei City 242; ${ }^{3}$ Department of Medical Imaging and Radiology, Shu-Zen Junior College of Medicine and Management, Kaohsiung 821; ${ }^{4}$ Department of Golden-Ager Industry Management, Chaoyang University of Technology, Taichung 413;

${ }^{5}$ Department of Orthopedic Surgery, Changhua Christian Hospital, Changhua 500-06;

${ }^{6}$ Department of Radiation Oncology, Cheng Hsien General Hospital, Bei-tou, Taipei 112;

${ }^{7}$ Department of Radiation Oncology, Taipei City Hospital, Renai Branch, Taipei 242;

${ }^{8}$ Department of Biomedical Imaging and Radiological Sciences, National Yang-Ming University, Taipei 112;

${ }^{9}$ Department of Radiation Oncology, Mackay Memorial Hospital, Taipei 104, Taiwan, R.O.C.
\end{abstract}

Received December 21, 2017; Accepted July 6, 2018

DOI: $10.3892 / o r .2018 .6568$

\begin{abstract}
Tetrandrine (TET), a traditional Chinese clinical agent, has been used for the treatment of many diseases, including cancers. The purpose of the present study was to investigate the combined effects of TET and ionizing radiation (IR) on murine CT26 colorectal adenocarcinoma cells in vitro and in vivo. A CT26 cell line transfected with dual HSV-1 thymidine kinase and firefly luciferase $(l u c)$ reporter genes was used. The half-maximal inhibitory concentration $\left(\mathrm{IC}_{50}\right)$ of TET in CT26/tk-luc cells was $\sim 10 \mu \mathrm{M}$. An additive effect was observed after combination of both agents based on a colony formation assay. Apoptosis and cleaved caspase- 3 levels were increased significantly in cells after combination treatment, as shown by flow cytometric analysis, DNA fragmentation and western blotting. However, tumor growth inhibition and therapeutic efficacy of TET combined with IR in vivo were identified to be synergistic, as monitored by tumor growth delay time, measured with a digital caliper. A
\end{abstract}

Correspondence to: Professor Jeng-Jong Hwang, Department of Biomedical Imaging and Radiological Sciences, National Yang-Ming University, No. 155, Section 2, Li-Nong Street, Bei-tou, Taipei 112, Taiwan, R.O.C.

E-mail: jjhwang@ym.edu.tw

Professor Yu-Jen Chen, Department of Radiation Oncology, Mackay Memorial Hospital, 92 North Chung Shan Road, Section 2, Taipei 104, Taiwan, R.O.C.

E-mail: chenmdphd@gmail.com

${ }^{*}$ Contributed equally

Key words: tetrandrine, radiation, synergism, luc, bioluminesence imaging, gamma scintigraphy significant inhibition of tumor growth was identified in the combination group compared with the radiation only group. Furthermore, non-invasive bioluminescent imaging (BLI) and gamma scintigraphy were also used to evaluate therapeutic efficacy. Both modalities revealed that the best tumor growth control was under combination treatment among all groups. The present study demonstrated that TET is not only beneficial for chemotherapy, but also has potential as a radiosensitizer for the treatment of cancer.

\section{Introduction}

Colorectal cancer (CRC) is the second leading cause of cancer-related deaths among all ages in the US, with nearly 130,000 new cases and 50,000 deaths in 2015 (1). The primary treatment modality for CRC is surgery. Radiotherapy (RT) may be used in combination with chemotherapy postoperatively to reduce the frequency of local recurrence (2). Adjuvant therapies have also been extensively studied due to the high incidence of postoperative recurrences. Concurrent chemoradiotherapy (CCRT) plays an important role in controlling the progression of CRC and in the palliation of CRC-related symptoms (3). Large portions of the small intestine, colorectum and urinary bladder are also included in the radiation field during RT with pelvic lymphatic drainage, thus limiting the delivery dose of RT. Therefore, radioprotectors such as amifostine and misoprostol have been used to improve therapeutic efficacy (4). Alternatively, radiosensitizers may be potential candidates for increasing the efficacy of RT for CRC.

The root of Stephania tetrandra has been used in traditional Chinese medicine for several decades to treat patients with arthritis, rheumatic disorders, silicosis, edema, inflammatory diseases and hypertension $(5,6)$. Tetrandrine (TET), a bisbenzylisoquinoline alkaloid isolated from the dried root of Hang-Fang-Chi (Stephania tetrandra S. Moore), possesses a number of medicinal properties, including proliferation, 
angiogenesis, migration and invasion. The cytotoxicity of TET may be via the induction of apoptosis and autophagy, the reversal of multidrug resistance and the enhancement of radiation sensitization (7-9). The antitumor effects of TET were demonstrated in several studies, including leukemia, lung carcinoma, hepatoblastoma, neuroblastoma and colorectal carcinomas (10-14). Furthermore, TET was revealed to enhance the radiosensitivity of human glioblastoma U138MG cells in vitro, suggesting its potential as an adjunct to RT (15). However, its potential in chemoradiotherapy of $\mathrm{CRC}$ in vivo remains to be elucidated.

Characteristics of apoptosis include loss of cellular contact with the matrix, cytoplasmic contraction, chromatin condensation, plasma membrane blebbing and DNA fragmentation (16). Both caspase- 8 and -3 , which are involved in the death receptor pathways, are considered to play important roles in TET-induced apoptosis $(17,18)$. IR can also act on the cellular membrane to generate ceramides via hydrolysis of sphingomyelin, resulting in apoptosis (19). TET has been reported to enhance the radiosensitivity of human esophageal carcinoma cells by arresting cells at G2/M, which are the most radiosensitive phases of the cell cycle (20). Here, we used a BALB/c CT26/tk-luc colorectal adenocarcinoma cell line and a tumor-bearing animal model to investigate the cytotoxic effects and therapeutic efficacy of TET alone and combined with IR in vitro and in vivo. In addition to digital caliper measurements for evaluating tumor growth, multiple modalities of molecular imaging, including bioluminescent imaging (BLI) and gamma scintigraphy, were used to monitor the inhibition of tumor growth

\section{Materials and methods}

Cell culture and transfection of HSV-1 thymidine kinase and luciferase genes. Standard cloning techniques were used to establish the pC1-tk-IRES-luc vector with dual reporter genes in the present study, as previously described (21). In brief, murine CT26 colorectal adenocarcinoma cells were cultured in RPMI-1640 medium (Invitrogen; Thermo Fisher Scientific, Inc., Waltham, MA, USA) supplemented with $10 \%$ fetal bovine serum (FBS; HyClone Laboratories; GE Healthcare Life Sciences, Logan, UT, USA). A plasmid vector carrying HSV-1 thymidine kinase $(t k)$ and luciferase $(l u c)$ genes was transfected into parental CT26 cells using Lipofectamine ${ }^{\mathrm{TM}} 2000$ transfection reagent (Invitrogen; Thermo Fisher Scientific, Inc.), and the cells were renamed CT26/tk-luc cells, as previously described (22). The CT26/tk-luc stable clones were cultured under the same condition as the parental cells. G418 $(600 \mu \mathrm{g} / \mathrm{ml})$ was added to the medium to maintain the stable expression of $t k$-luc genes.

TET preparation. For the in vitro study, TET (cat. no. 365629; Sigma-Aldrich; Merck, St. Louis, MO, USA) was dissolved in dimethyl sulfoxide (DMSO) to $10 \mathrm{mM}$, sterilized by filtration through a $0.22-\mu \mathrm{m}$ filter as a stock solution, then stored at $-20^{\circ} \mathrm{C}$. For the working solution, the stock solution was diluted to desired concentrations with serum-free medium immediately before each experiment. The final concentration of DMSO was $<0.5 \%$. For the in vivo study, TET was dissolved in a drop of $1 \mathrm{~N} \mathrm{HCl}$ and then adjusted to $\mathrm{pH} 7.0$ with $1 \mathrm{~N}$
$\mathrm{NaOH}$, then further diluted with $0.9 \% \mathrm{NaCl}$ solution to desired concentrations. Finally, it was sterilized by filtration through a $0.22-\mu \mathrm{m}$ filter and stored at $4^{\circ} \mathrm{C}$.

Irradiation. For the in vitro study, cultured monolayer cells were irradiated with a Co-60 AECL Eldorado-78 irradiator at a dose rate of $32 \mathrm{cGy} / \mathrm{min}$ at room temperature, $80 \mathrm{~cm}$ source-to-surface distance $(\mathrm{SSD})$ and a field size of $30 \times 30 \mathrm{~cm}$. Before and immediately after irradiation, cells were maintained on ice to arrest the cell cycle. For the tumor-bearing animal model, 6 mice per group were placed in an acrylic restraint and irradiated with $18 \mathrm{~Gy}$ (field size of 30x5 cm) administered in 6 fractions, one fraction a day, three times a week.

Clonogenic survival assay. CT26/tk-luc cells were seeded in T-25 flasks $\left(1 \times 10^{6}\right.$ cells/flask) overnight, then treated with $0,4,8,16,24$ and $32 \mu \mathrm{M}$ of TET for $3 \mathrm{~h}$, different doses of radiation, or $20 \mu \mathrm{M}$ TET for $3 \mathrm{~h}$ followed by irradiation. The plating efficiency (PE) was defined as the number of colonies divided by the number of cells plated in the control group. The surviving fraction (SF) under various treatments was the number of counted colonies divided by the number of colonies plated and corrected for PE. The mean lethal dose $\left(D_{0}\right)$ was calculated from the radiation survival curve using $\mathrm{D}_{10}=$ $2.3 \mathrm{xD}_{0}$. The experiments were repeated more than three times.

Sub-G1 population assayed by flow cytometric analysis. CT26/tk-luc cells were cultured in $60 \mathrm{~mm}$-diameter dishes ( $1 \times 10^{6}$ cells/dish) overnight. When the cell growth reached $\sim 80 \%$ confluence, the cells were treated with different concentrations of TET for $3 \mathrm{~h}$, or TET for $3 \mathrm{~h}$ followed by $4 \mathrm{~Gy}$ irradiation. Cells were washed twice with phosphate-buffered saline (PBS), then cultured in fresh medium containing $10 \%$ FBS for a further $24 \mathrm{~h}$ prior to analysis with a FACScan flow cytometer (BD Biosciences, Franklin Lakes, NJ, USA). Cells in the sub-G1 phase (i.e. apoptotic cells) could be distinguished from cells with the normal diploid DNA peak $\left(\mathrm{G}_{0} / \mathrm{G}_{1}\right.$ phase $)$ on the fluorescence profiles of propidium iodide-stained cells. The percentage of cells in the sub-G1 phase was estimated with CellQuest software (BD Biosciences). CT26/tk-luc cells treated with $50 \mu \mathrm{M}$ camptothecin (CAM; cat. no. 7689-03-4; Sigma-Aldrich; Merck KGaA) for $24 \mathrm{~h}$ were used as the positive control.

DNA fragmentation assay. CT26/tk-luc cells were cultured in $60 \mathrm{~mm}$-diameter dishes $\left(1 \times 10^{6}\right.$ cells/dish $)$ and incubated at $37^{\circ} \mathrm{C}$ overnight, then treated with $0,10,20$ and $50 \mu \mathrm{M}$ TET for $3 \mathrm{~h}$. The combination group was irradiated with 4 Gy immediately after TET treatment. Cells were harvested and centrifuged at $80 \mathrm{x} \mathrm{g}$ for $5 \mathrm{~min}$ and supernatants were aspirated. DNA fragmentation was performed using Suicide Track $^{\mathrm{TM}}$ DNA Ladder Isolation kit [cat. no. AM41; Calbiochem; EMD Millipore (Billerica, MA, USA)]. Total DNA was extracted using the kit, then dissolved in $50 \mu \mathrm{l}$ resuspension buffer. A volume of $21 \mu$ l DNA sample was transferred to a clean centrifuge tube and $4 \mu \mathrm{l}$ of $6 \mathrm{X}$ gel loading buffer was added. Then, it was loaded onto a $1.5 \%$ agarose gel and stained with ethidium bromide for electrophoresis in 0.5 M Tris/EDTA buffer. 
Total protein isolation. CT26/tk-luc cells were harvested at $24 \mathrm{~h}$ after TET alone and combined with $4 \mathrm{~Gy}$ irradiation, then washed twice with PBS and centrifuged at $80 \mathrm{x} g$ for $3 \mathrm{~min}$. The cell pellet was resuspended in $100 \mu \mathrm{l}$ protein lysis buffer (for $1.5 \times 10^{6}-2 \times 10^{7}$ ). Suspended cells were transferred to an Eppendorf tube on ice, then centrifuged at 10,956 $\mathrm{x}$ g for $10 \mathrm{~min}$ at $4^{\circ} \mathrm{C}$. The supernatant was transferred to a new tube on ice and the pellet was discarded. The total protein concentration was determined using bovine serum albumin (BSA) as the standard by measuring the absorbance at $595 \mathrm{~nm}$. Samples were stored at $-20^{\circ} \mathrm{C}$ for western blot analysis and caspase-3 activity assay.

Western blotting. Lysis buffer $(50 \mathrm{mM}$ Tris- $\mathrm{HCl}, \mathrm{pH} 8.0$, $120 \mathrm{mM} \mathrm{NaCl}, 0.5 \% \mathrm{NP}-40$ and $1 \mathrm{mM}$ phenylmethanesulfonyl fluoride) was used for the extraction of total proteins from cells of each group at $4^{\circ} \mathrm{C}$. The proteins $(40 \mu \mathrm{g} / \mathrm{lane})$ were separated via $10 \%$ SDS-PAGE and transferred onto a polyvinylidene difluoride (PVDF) membrane (EMD Millipore, Billerica, MA, USA). The membrane was blocked with $5 \%$ non-fat milk in TBST buffer solution (10 mM Tris-base, $150 \mathrm{mM} \mathrm{NaCl}, 0.1 \%$ Tween-20) for $1 \mathrm{~h}$ at room temperature, followed by incubation with the appropriate primary antibodies against caspase-3 (1:300; cat. no. IMG-144A; Imgenex, Novus Biologicals, LLC, Littleton, CO, USA), $\beta$-actin (1:3,000; cat. no. MAB1501; Chemicon International, Inc., Billerica, MA, USA) overnight at $4^{\circ} \mathrm{C}$. The membranes was further incubated with anti-mouse IgG-horseradish peroxidase (HRP) for $1 \mathrm{~h}$ at room temperature (1:5,000; cat. no. AP124P; Chemicon International, Inc.), then detected using ECL Western Blot Chemiluminescence Reagent Plus (cat. nos. NEL104 and NEL105; PerkinElmer Life Science, Inc., Waltham, MA, USA). Membranes were dried by dabbing with tissue, then exposed to FUJI Medical X-ray film (cat. no. 50407; Fujifilm, Tokyo, Japan) and quantified with Scion Image software (version 4.0.3.2; Scion Corp., Frederick, MD, USA).

Caspase-3 activity analysis. Caspase-3 plays an important role in triggering the apoptotic process, and its activity has been suggested to be an index of apoptosis (23). To determine whether caspase activation was involved in TET-induced cell death, CT26/tk-luc cells were exposed to TET for $3 \mathrm{~h}$ with or without radiation, and incubated for a further 3,9 and $21 \mathrm{~h}$ at $37^{\circ} \mathrm{C}$. The protein expression of caspase- 3 was assayed by western blotting. Total protein $(50 \mu \mathrm{g})$ was added to the buffer to a final volume of $500 \mu \mathrm{l}$. Ac-DEVD-AFC $(20 \mu \mathrm{M})$, the substrate for caspase-3, was added to the sample and incubated at $37^{\circ} \mathrm{C}$ for $30 \mathrm{~min}$. After cleavage by activated caspase- 3 , the substrate released a yellow-green fluorescent compound, AFC, which could be detected with a spectrophotometer (Hitachi F-4500; Hitachi, Ltd., Tokyo, Japan), with excitation and emission at 380 and $508 \mathrm{~nm}$, respectively. Cells treated with $50 \mu \mathrm{M}$ $\mathrm{CAM}$ for $24 \mathrm{~h}$ were used as the positive control. The results were expressed as the percentage change in activity compared with the untreated control. The experiments were performed three times independently.

Tumor-bearing animal model. Six-week-old male BALB/c mice (number of mice, 85 ; body weight, $20 \pm 2 \mathrm{~g}$ ) were housed in the following conditions: $22 \pm 1^{\circ} \mathrm{C}, 55-60 \%$ relative humidity, 12-h light/dark cycle control and food and water with ad libitum. The mice were anesthetized with an i.p. injection of $45 \mathrm{mg} / \mathrm{kg}$ ketamine and $15 \mathrm{mg} / \mathrm{kg}$ xylazine, and $2 \times 10^{5} \mathrm{CT} 26 / t k$-luc cells in $0.2 \mathrm{ml}$ serum-free medium were subcutaneously injected into the right hind legs. Perpendicular tumor diameters were measured using a digital caliper and the tumor volume was calculated using the formula: $0.523 \mathrm{x}$ (length $\mathrm{x}$ width $\mathrm{x}$ thickness) (24) Treatment was initiated when the tumor volume reached $100 \mathrm{~mm}^{3}$. Mice were randomly separated into six groups (6 mice for the control group, 7 mice per group for the rest groups): Normal saline, $5 \mathrm{mg} / \mathrm{kg}$ TET for 12 consecutive days, $10 \mathrm{mg} / \mathrm{kg}$ TET (three times a week for 2 weeks), $20 \mathrm{mg} / \mathrm{kg}$ TET (three times in 2 weeks), radiation alone (3 Gy/fraction, three fractions per week for 2 weeks) and concurrent $10 \mathrm{mg} / \mathrm{kg}$ TET and 3 Gy radiation. Normal saline and TET were i.p. injected. Tumor growth inhibition was measured by a digital caliper, non-invasive BLI and gamma scintigraphy. All animal experiments were repeated twice.

Tumor growth inhibition assay. Body weight and tumor volume were assayed twice per week throughout the experimental period. The time required to reach 8 -fold of the initial tumor volume (i.e. $100 \mathrm{~mm}^{3}$ ) was used as the biological endpoint. Tumor growth delay (TGD) was calculated as follows: TGD $=\mathrm{TGT}_{\text {treated }} \mathrm{TGT}_{\text {control }}$, where TGT represents tumor growth time (25). The enhancement ratio (ER) was calculated as follows: $\mathrm{ER}=\left(\mathrm{TGD}_{\mathrm{TET}+\mathrm{IR}}-\mathrm{TGD}_{\mathrm{IR}}\right) / \mathrm{TGD}_{\mathrm{TET}}$, where $\mathrm{TGD}_{\mathrm{TET}+\mathrm{IR}}$ stands for the tumor growth delay of the combination group; $\mathrm{TGD}_{\mathrm{IR}}$ stands for the tumor growth delay of the radiation alone group and $\mathrm{TGD}_{\mathrm{TET}}$ represents the tumor growth delay of the TET alone group.

In vivo survival curve. Survival curves were determined by the Kaplan-Meier method. Mice were considered expired when the tumor volume reached $2,500 \mathrm{~mm}^{3}$ post-treatment in accordance with the Institutional Animal Care and Use Committee regulations (26). IACUC approval no. for the animal experiments in the present study was 950501 issued by the National Yang-Ming University.

Bioluminescent imaging (BLI). CT26/tk-luc murine CRC cells $\left(2 \times 10^{5}\right.$ cells) were inoculated into the flank of BALB/c mice to monitor tumor growth and the therapeutic efficacy of TET with or without radiation, assayed by direct measurement with calipers and BLI using the Xenogen IVIS50 Imaging system (Xenogen Corp., Alameda, CA, USA). Images and measurements of bioluminescent signals were acquired and analyzed using Living Image software (Xenogen Corp.). Mice were i.p. injected with D-luciferin $150 \mathrm{mg} / \mathrm{kg}$ in PBS and anesthetized using 1-3\% isoflurane $15 \mathrm{~min}$ prior to imaging. Mice were placed on a warmed platform inside the camera box and received continuous exposure to $1-2 \%$ isoflurane to sustain sedation during imaging. The image acquisition time ranged from $30 \mathrm{sec}$ to 5 min depending on the bioluminescence of the tumors. Regions of interest (ROIs) from displayed images were drawn around the tumor and quantified as photons/second ( $\mathrm{ph} / \mathrm{s})$ using the Living Image software (version 3.1; Information Technologies LLC, St. Louis, MO, USA). The serial bioluminescent signals were quantified and displayed over time. 

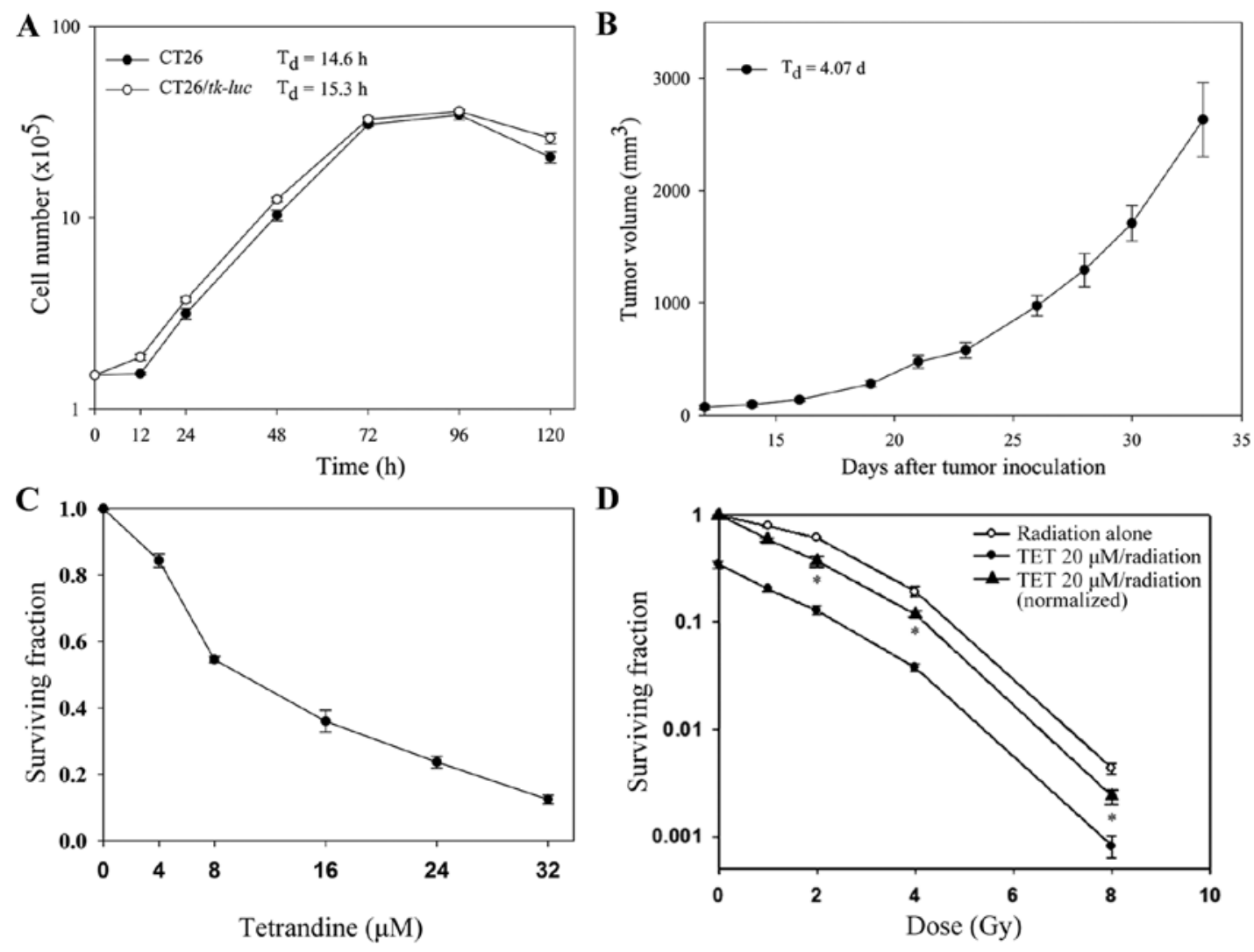

Figure 1. Cytotoxicity and survival of CT26/tk-luc cells treated with tetrandrine (TET) and/or radiation. (A) Growth curves of CT26 and CT26/tk-luc cells in vitro. The cell doubling times were 14.6 and $15.3 \mathrm{~h}$, respectively. (B) Tumor growth curve of C26/tk-luc tumor-bearing mice. The tumor doubling time was 4.07 days. (C) Cytotoxicity of TET in CT26/tk-luc cells. CT26/tk-luc cells were treated with various concentrations of TET for $3 \mathrm{~h}$, then evaluated by clonogenic assay to determine the surviving fractions. (D) Survival curves of CT26/tk-luc cells treated with TET and radiation. CT26/tk-luc were treated with various radiation doses alone and $20 \mu \mathrm{M}$ TET for $3 \mathrm{~h}$, then combined with various radiation doses, and evaluated by clonogenic assay. The survival curve for radiation treatment alone is shown by $(\circ)$, while combination treatment is shown by $(\bullet)$. Normalization of this survival curve is shown by $(\mathbf{\Lambda})$. Data are expressed as the mean \pm standard error $(" \mathrm{P}<0.05)$.

Gamma scintigraphy. Planar imaging was performed on $\mathrm{BALB} / \mathrm{c}$ mice bearing CT26/tk-luc tumors derived from $2 \times 10^{5}$ cells as aforementioned. After mice were injected with $100 \mu \mathrm{Ci}\left[{ }^{131} \mathrm{I}\right]$ FIAU via the tail vein, static imaging was obtained from anaesthetized animals at $24 \mathrm{~h}$ post-injection with e.Cam Multiangle Cardiac camera (Siemens AG, Munich, Germany) equipped with a pinhole collimator. ROIs were selected over the tumor area and the reference organs.

Statistical analysis. All data are presented as the mean \pm standard error (SE). Student's t-test was used for comparison between two groups. The differences among multiple groups was analyzed using ANOVA and the Tukey-Kramer method for the post hoc test. A difference between the means was considered significant at $\mathrm{P}<0.05$.

\section{Results}

Cytotoxicity of TET and radiation in CT26/tk-luc cells. The cell growth curves of CT26 and CT26/tk-luc in vitro are shown in Fig. 1A. The cell doubling time of CT26 and CT26/tk-luc were 14.6 and $15.3 \mathrm{~h}$, respectively. Fig. 1B shows the tumor growth curve of C26/tk-luc in vivo. The tumor doubling time of CT26/tk-luc-bearing mice was 4.07 days. To evaluate the cytotoxicities of TET and radiation, surviving fractions of
CT26/tk-luc cells were determined by colony formation assay. Cells were treated with various concentrations of TET for $3 \mathrm{~h}$. The growth inhibition by TET was found to occur in a dose-dependent manner. The $\mathrm{IC}_{50}(50 \%$ inhibition concentration) was $\sim 10 \mu \mathrm{M}$, as shown in Fig. 1C. The radiation response of CT26/tk-luc cells was examined following single doses of Co-60 gamma irradiation. The radiation survival curve is shown in Fig. 1D. The $\mathrm{D}_{0}$ was $\sim 2.0 \mathrm{~Gy}$, as calculated from the formula $\mathrm{D}_{10}=2.3 \mathrm{D}_{0}$ using $\mathrm{D}_{10}=4.7 \mathrm{~Gy}$. In addition, CT26/tk-luc cells were treated with $20 \mu \mathrm{M}$ TET for $3 \mathrm{~h}$, and then combined with different doses of radiation. The survival curve for the combination of TET and radiation shifted the radiation survival curve downward without changing the curve shape, suggesting that the combined effect of TET and radiation on CT26/tk-luc cells was additive.

Analysis of the sub-G1 fraction by flow cytometry and apoptosis by DNA fragmentation assay. Apoptosis induced by TET with or without radiation in CT26/tk-luc cells was also determined via the sub-G1 cell population by flow cytometry. Fig. 2A reveals the DNA histograms of CT26/tk-luc cells post-treatment. The sub-G1 populations of CT26/tk-luc cells were $0.93,0.95,8.09$ and $55.76 \%$ for $0,10,20$ and $50 \mu \mathrm{M}$, respectively, following TET treatment for $3 \mathrm{~h}$. The sub-G1 populations of CT26/tk-luc cells were $0.85,1.06,11.72$ and 
A
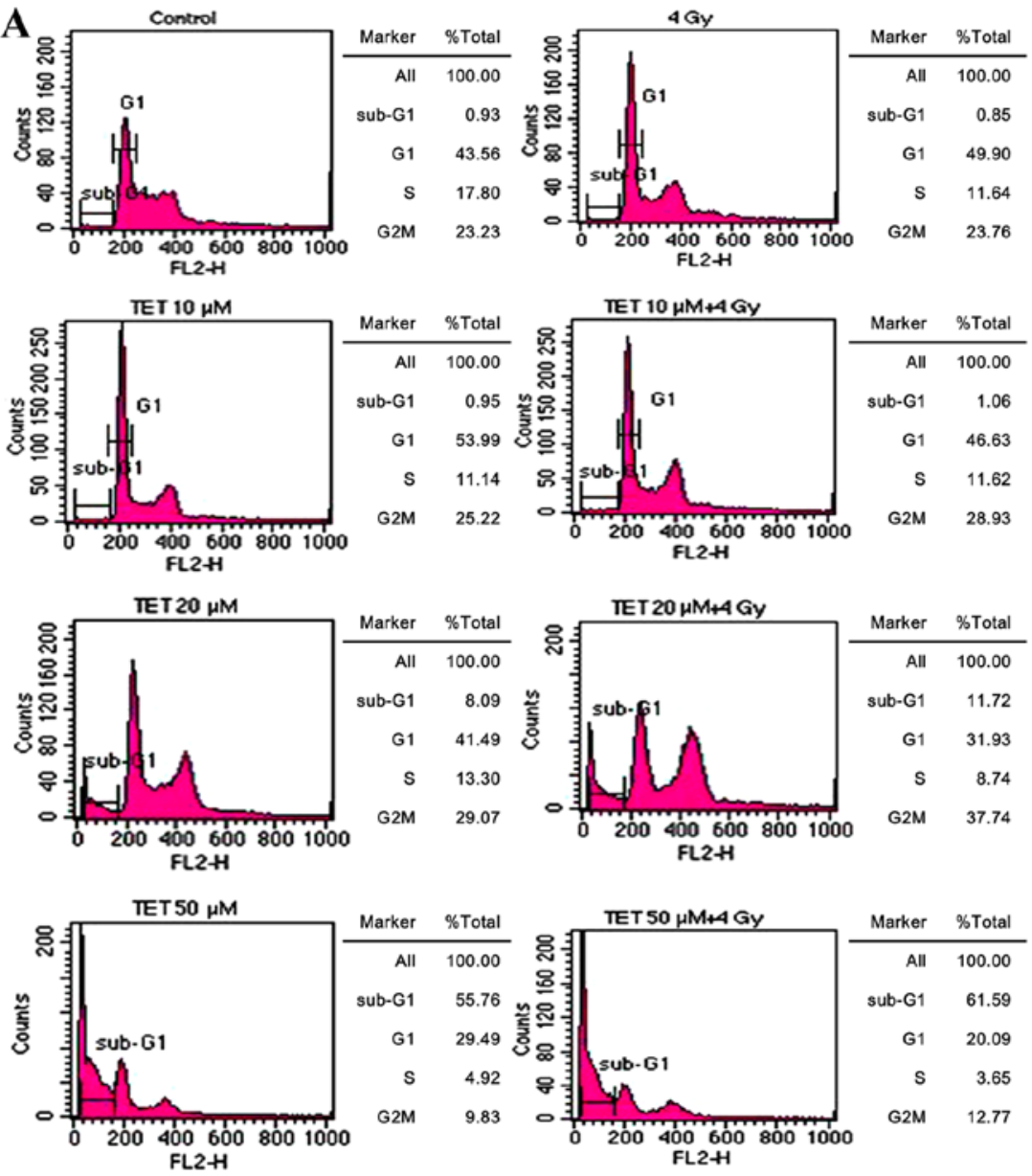
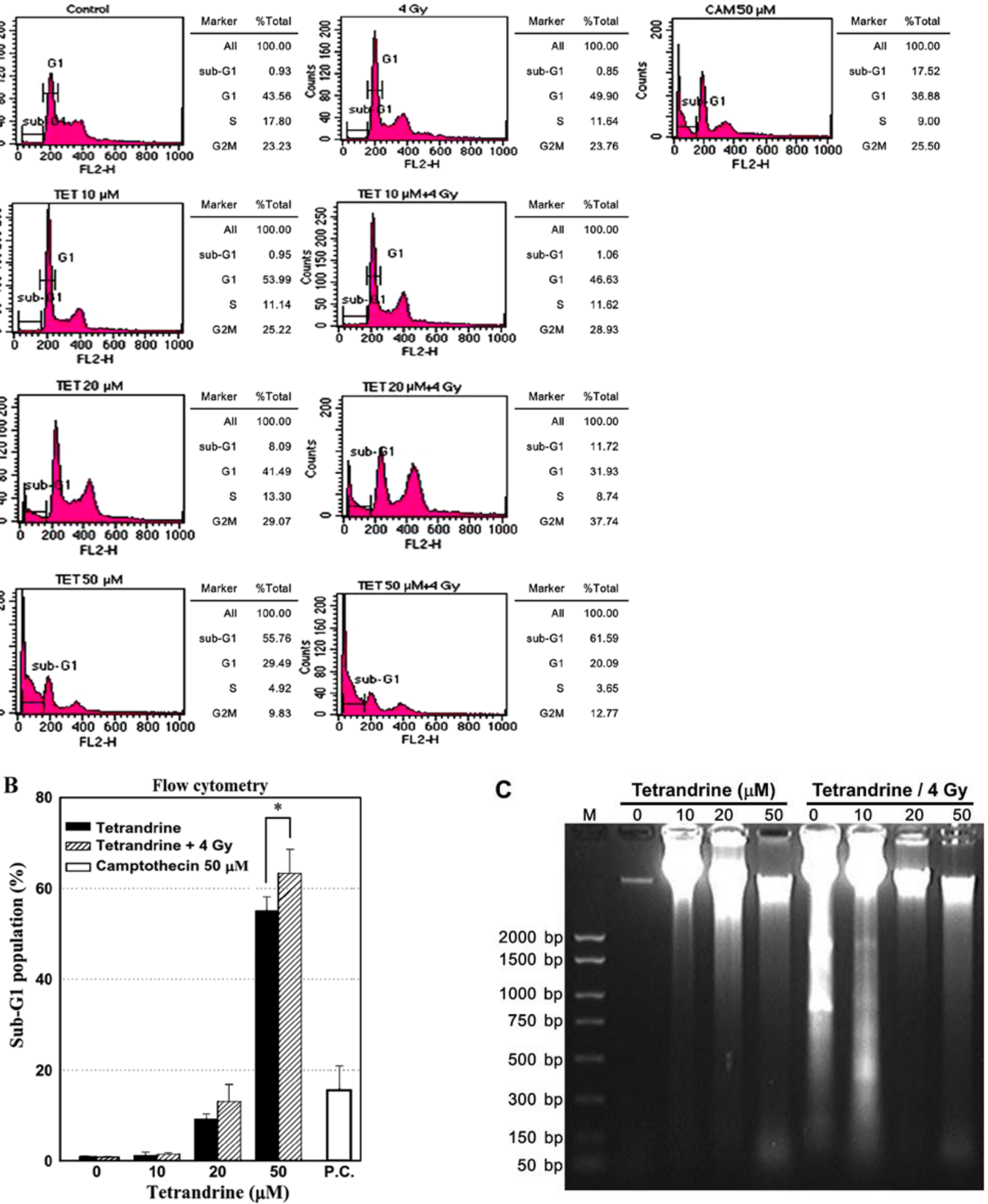

Figure 2. Cell cycle analysis of CT26/tk-luc cells treated with tetrandrine (TET) and/or radiation. Cells were treated with various concentrations of TET alone for $3 \mathrm{~h}$ or combined with $4 \mathrm{~Gy}$ of radiation. DNA content was evaluated by (A) propidium iodide staining and (B) analyzed with a FACSCalibur flow cytometer. Camptothecin (CAM; $50 \mu \mathrm{M}$ ) was used as the positive control (P.C.). Representative results are presented. Sub-G1 populations obtained from three independent experiments were quantified to identify the percentage of apoptotic cells. (C) Agarose gel electrophoresis of DNA extracted from CT26/tk-luc cells treated with various concentrations of TET alone or combined with $4 \mathrm{~Gy}$ of radiation. DNA fragmentation was more evident in the combination group. M, marker. The numbers on the $y$-axis stand for base pairs. ${ }^{*} \mathrm{P}<0.05$.

$61.59 \%$ for $0,10,20$ and $50 \mu \mathrm{M}$ TET treatment for $3 \mathrm{~h}$ combined with 4 Gy, respectively. Notably, significant G1 phase arrest was found in all TET-treated groups. CAM $(50 \mu \mathrm{M})$ was used as the positive control. The mean sub-G1 populations in CT26/tk-luc cells induced by TET with or without radiation determined by flow cytometric assay are displayed in Fig. 2B. The decrease in cell viability due to apoptosis rather than necrosis was detected by DNA ladder assay. To evaluate the nature of TET-induced apoptosis, CT26/tk-luc cells were treated with the indicated TET concentrations or treated with 

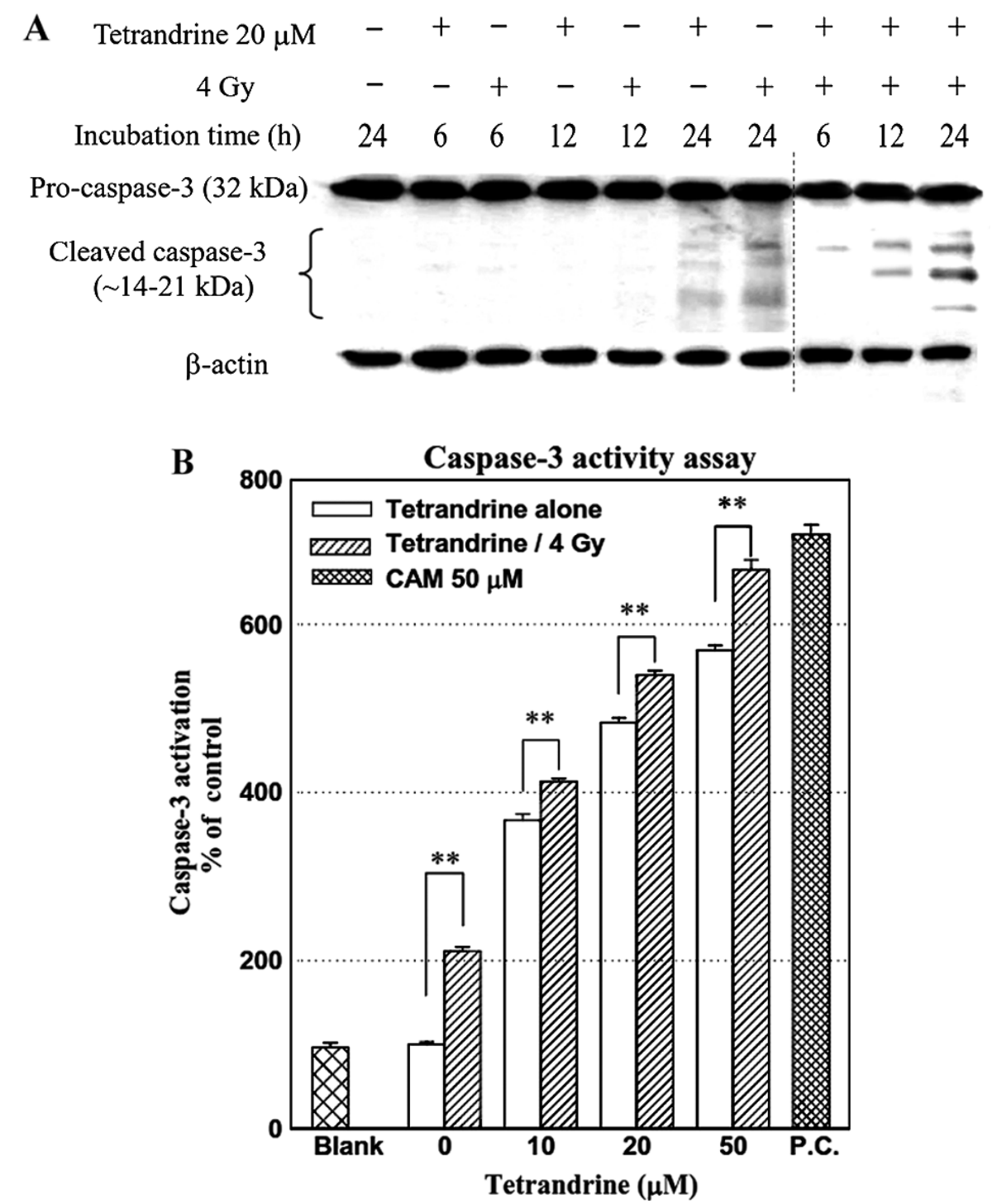

Figure 3. Effect of tetrandrine on caspase-3 activation with or without radiation in CT26/tk-luc cells. (A) Cells were treated with $20 \mu \mathrm{M}$ tetrandrine for $3 \mathrm{~h}$ with or without $4 \mathrm{~Gy}$ irradiation, then they were incubated for a further 3,9 and $21 \mathrm{~h}$, followed by western blot analysis for both pro- and cleaved caspase-3. A 10-column gel was used to perform the experiment. In order to include the markers, it was necessary to use two separate gels. The dotted line between lanes 7 and 8 indicates that the result was obtained from two separated gels with the samples from the same experiment. (B) CT26/tk-luc cells were treated with 0 , 10,20 and $50 \mu \mathrm{M}$ tetrandrine with or without $4 \mathrm{~Gy}$ for $24 \mathrm{~h}$ followed by western blot analysis and the results were quantified. The activation of caspase-3 was compared between TET alone and the combination treatment groups. Camptothecin (CAM; $50 \mu \mathrm{M})$ was used as the positive control (P.C.). Data are presented as the mean \pm standard error $\left({ }^{* *} \mathrm{P}<0.01\right)$.

TET in combination with radiation. Internucleosomal DNA fragmentation was observed, as revealed in Fig. 2C. The results indicated that TET alone or in combination with radiation induced apoptosis in CT26/tk-luc cells.

Western blotting for caspase-3 activity assay. Activation of caspase- 3 can be demonstrated from the cleavage of pro-caspase- 3 into cleaved caspase- 3 . Cleaved caspase- 3 was detected in CT26/tk-luc cells after $20 \mathrm{mM}$ TET treatment for 6,12 or $24 \mathrm{~h}$ with or without 4 Gy irradiation, respectively, as shown in Fig. 3A. In addition, CT26/tk-luc cells were treated with various concentrations of TET for $24 \mathrm{~h}$ with or without 4 Gy. The expression levels of cleaved caspase-3 quantified by Scion imaging software were higher when concurrently treated with TET and radiation, as shown in Fig. 3B. These results unambiguously indicated that activation of caspase- 3 contributed to TET-induced apoptosis in murine colorectal CT26/tk-luc cancer cells.

TET combined with radiation enhances the regression of CT26/tk-luc tumors in vivo. Antitumor activity of TET combined with radiation was assessed in a CT26/tk-luc tumor-bearing Balb/c mouse model. The experimental protocol is presented in Fig. 4A. The monitoring of CT26/tk-luc tumor volumes was initiated on day 12 post-tumor cell inoculation, when bulges were observed. Treatment was administered when the tumor volume reached $\sim 100 \mathrm{~mm}^{3}$. The tumor growth inhibition caused by TET with or without irradiation is shown in Fig. 4B. No significant differences were observed among the groups treated with TET alone in any fractionation protocol. However, radiation alone and combination therapy exhibited a significant decrease in tumor volume as compared 
Table I. Therapeutic efficacy of TET with or without RT on CT26/tk-luc tumor growth.

\begin{tabular}{|c|c|c|c|}
\hline Groups & No. of mice & Tumor growth time ${ }^{a}$ (days \pm SE) & Tumor growth delay time ${ }^{\mathrm{b}}($ days $\pm \mathrm{SE})$ \\
\hline Control & 6 & $8.0 \pm 1.8$ & $0.0 \pm 0.0$ \\
\hline TET $5 \mathrm{mg} / \mathrm{kg}$ x 12 & 7 & $10.7 \pm 1.6$ & $2.7 \pm 2.4$ \\
\hline TET $10 \mathrm{mg} / \mathrm{kg}$ x 6 & 7 & $10.7 \pm 1.0$ & $2.7 \pm 2.1$ \\
\hline TET $20 \mathrm{mg} / \mathrm{kg}$ x 3 & 7 & $11.3 \pm 1.8$ & $3.3 \pm 2.5$ \\
\hline RT (3 Gy x 6) & 7 & $18.4 \pm 2.1$ & $10.4 \pm 2.8$ \\
\hline TET $10 \mathrm{mg} / \mathrm{kg}+\mathrm{RT}$ & 7 & $23.1 \pm 1.3$ & $15.1 \pm 2.2$ \\
\hline
\end{tabular}

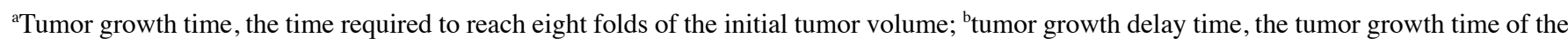
treated group minus that of the control group. TET, tetrandrine; RT, radiotherapy.
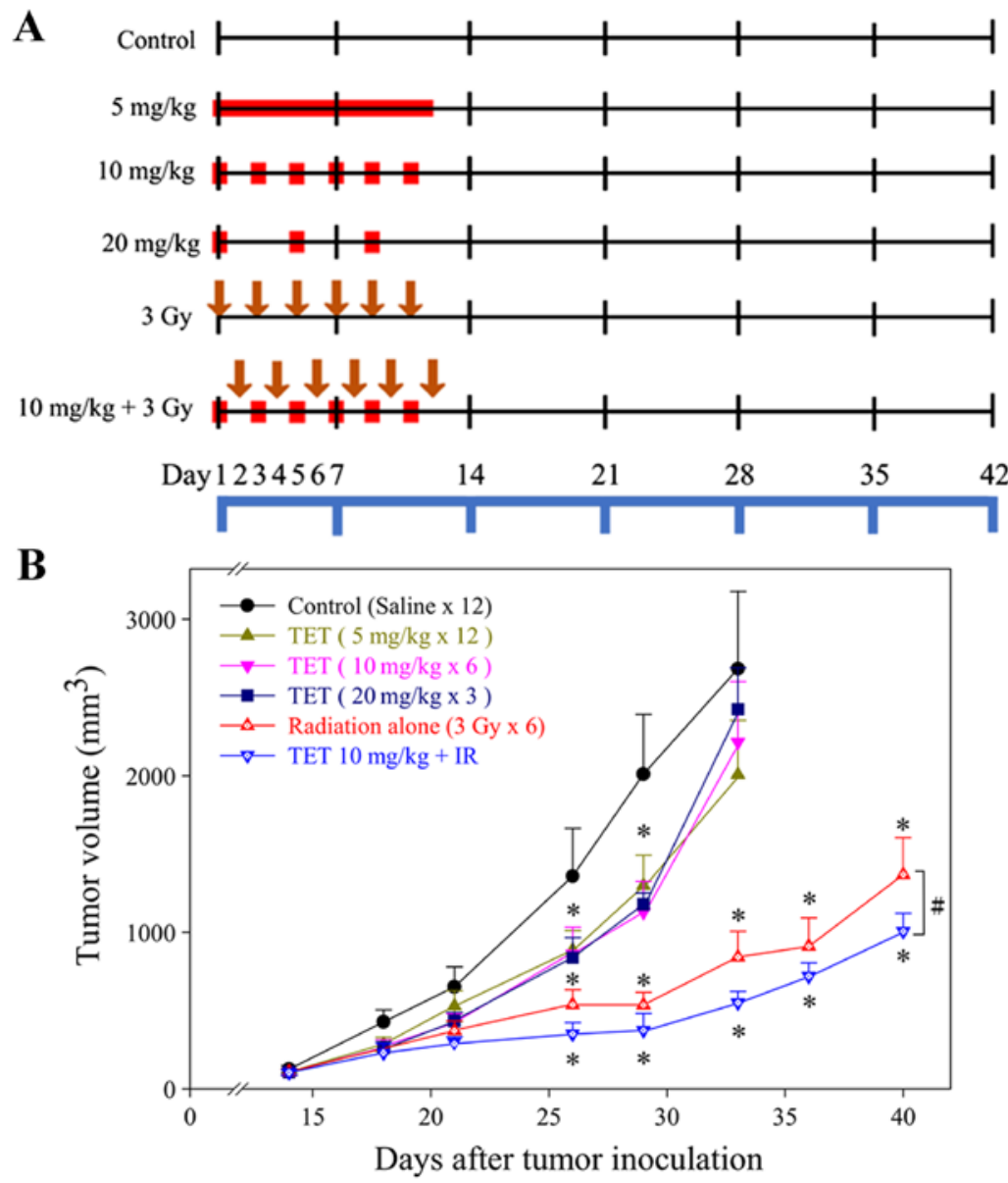

Figure 4. Tumor growth curves in CT26/tk-luc tumor-bearing mice. (A) Experimental design for the evaluation of therapeutic efficacy of tetrandrine (TET). Treatment was initiated when the tumor volume reached $100 \mathrm{~mm}^{3}$. Mice were randomly assigned to six groups: Control, 20,10 and $5 \mathrm{mg} / \mathrm{kg}$ TET, radiation alone and combination. TET was administered every 4 days for 2 weeks in the $20 \mathrm{mg} / \mathrm{kg}$ group, every 2 days for 2 weeks in the $10 \mathrm{mg} / \mathrm{kg}$ group and for 12 consecutive days in the $5 \mathrm{mg} / \mathrm{kg}$ group by intraperitoneal injection (i.p.), i.e. 3, 6 and 12 fractions for a total of $60 \mathrm{mg} / \mathrm{kg}$. For the radiation alone group, 3 Gy was administered every other day in 6 fractions, and for the combination group, $10 \mathrm{mg} / \mathrm{kg}$ TET or 3 Gy were alternately administered every day for 2 weeks. The red symbols indicate the time points for the administration of TET, while the downward brown arrows indicate irradiation. (B) Tumor growth curves of CT26/tk-luc tumor-bearing mice following different treatments. The time required to reach an 8 -fold increase from the initial tumor volume was used as the endpoint for determining the tumor growth delay assays. $n=7$ for all mouse groups, except $n=6$ in the control group. ${ }^{*} \mathrm{P}<0.05$ vs. the control, and ${ }^{\#} \mathrm{P}<0.05$, the radiation alone group vs. the combination group.

with either TET alone or the control group $(\mathrm{P}<0.01)$. To determine whether TET could enhance tumor growth inhibition in irradiated tumors, mice were i.p. injected with $10 \mathrm{mg} / \mathrm{kg}$ TET $24 \mathrm{~h}$ before 3 Gy irradiation for a total of six administrations. The time required to reach $800 \mathrm{~mm}^{3}$ tumor volume for the control, 5,10 and $20 \mathrm{mg} / \mathrm{kg}$ of TET, radiation alone (3 Gyx6) and combination therapy (TET $10 \mathrm{mg} / \mathrm{kg}+3 \mathrm{~Gy}$ ) groups was $8.0 \pm 1.8,10.7 \pm 1.6,10.7 \pm 1.0,11.3 \pm 1.8,18.4 \pm 2.1$ and $23.1 \pm 1.3$ days, respectively. These results are presented in Table I. The TGD times were $2.7 \pm 2.4,2.7 \pm 2.1,3.3 \pm 2.5$, 


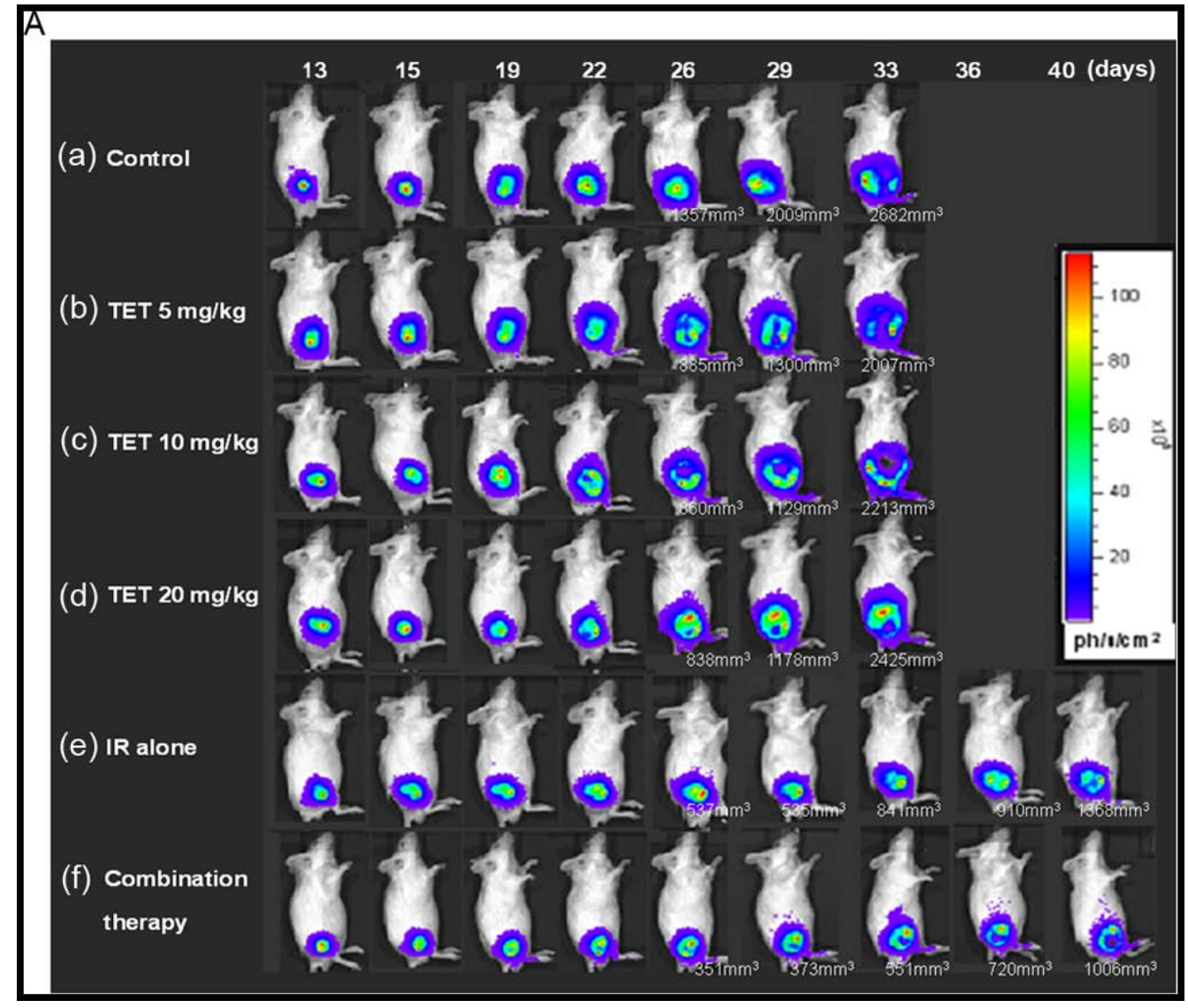

B
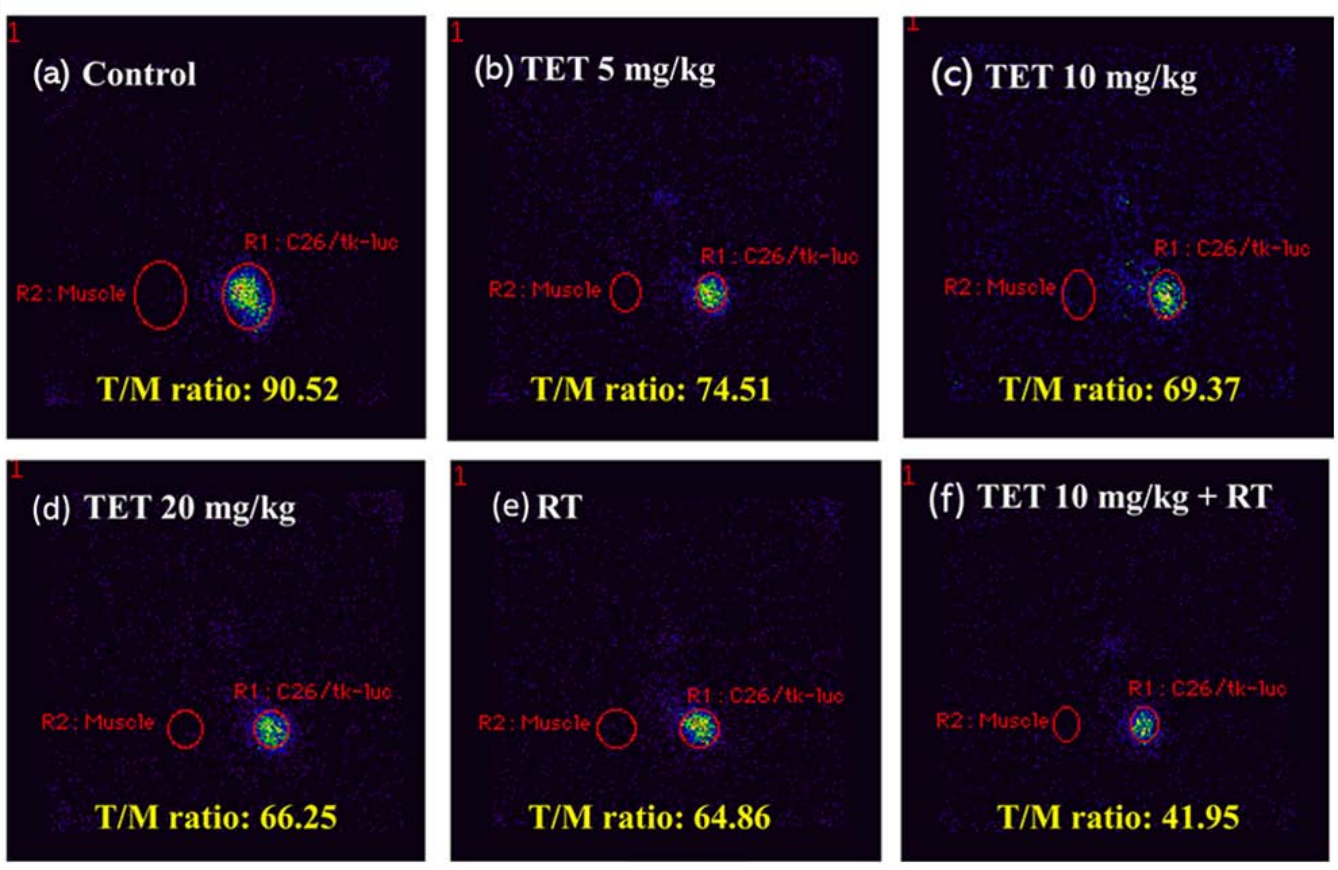

Figure 5. Growth of CT26/tk-luc tumors in mice was monitored with bioluminescent imaging (BLI) and gamma scintigraphy. (A) The CT26/tk-luc tumor growth of a representative mouse in each group was assayed with BLI using the Xenogen IVIS50 system. (B) Gamma scintigraphy of CT26/tk-luc tumors in mice was performed at $24 \mathrm{~h}$ following $100 \mu \mathrm{Ci} / 100 \mathrm{ml}{ }^{131}$ I-FIAU intravenous administration via the tail vein. The regions-of-interest (ROIs) of the tumors exhibited higher radioactivity compared with their normal counterparts, as illustrated by the tumor/muscle (T/M) ratios, which were the lowest in the combination group. a, control; b, TET 5 mg/kg x 12; c, TET 10 mg/kg x 6; d, TET 20 mg/kg x 3; e, IR (RT 3Gy x6); f, TET 10 mg/kg x 6 + RT 3 Gy x 6 .

$10.4 \pm 2.8$ and $15.1 \pm 2.2$ for 5,10 and $20 \mathrm{mg} / \mathrm{kg}$ of TET, radiation alone and combination therapy, respectively. The calculated ER for the combination group compared with the radiation alone group based on the TGD time was 1.45 , indicating that TET combined with IR for the treatment of CT26/tk-luc cancer in vivo was synergistic. 

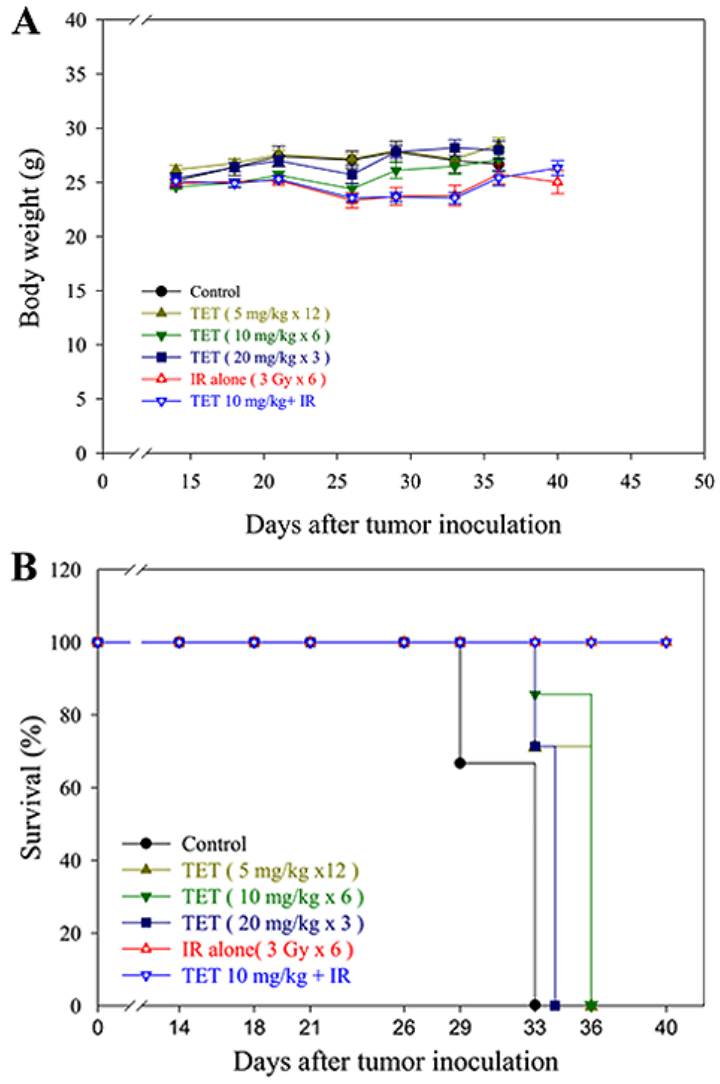

Figure 6. Body weight and overall survival of mice were monitored throughout the study period. (A) The body weight change was $\pm 10 \%$ for each group throughout the study period $(\mathrm{P}=0.4)$, suggesting no general toxicity of the treatments. (B) Kaplan-Meier survival analysis of BALB/c mice implanted with CT26/tk-luc colorectal adenocarcinoma cells. Mice were considered expired when the tumor volume reached $2,500 \mathrm{~mm}^{3}$ during the study period. At the end of the experiment (day 40), the survival of the control group and 5, 10 and $20 \mathrm{mg} / \mathrm{kg}$ tetrandrine (TET) groups were all expired. All those treated with radiation alone and combination therapy survived.

Evaluation of therapeutic efficacy with BLI and gamma scintigraphy. CT26/tk-luc cells $\left(2 \times 10^{5}\right)$ were inoculated into the right flank of BALB/c mice to monitor subcutaneous tumor growth and the therapeutic efficacy of TET with or without radiation by non-invasive molecular imaging modalities: BLI using the Xenogen IVIS50 system and gamma scintigraphy. Fig. 5A shows the longitudinal monitoring of the tumor growth by BLI. In addition, the mice were also monitored by gamma scintigraphy at $24 \mathrm{~h}$ after ${ }^{131}$ I-FIAU intravenous administrations. The representative images of the CT26/tk-luc tumor-bearing animals are shown in Fig. 5B. ROIs of the tumor exhibited higher radioactivity compared with normal soft tissue. The radioactive counts over the tumor area were normalized against the tumor size (pixels). The ROI was also created over a non-tumor area (muscle) to determine the radioactivity of the background. Notably, the tumor/muscle (T/M) ratios were decreased as the therapeutic time increased. The tumor/muscle (T/M) ratios were 90.52, 74.51, 69.37, 66.25, 64.86 and 41.95 for the control, 5,10 or $20 \mathrm{mg} / \mathrm{kg}$ TET, radiation alone and combination therapy, respectively.

Body weight and survival curves in vivo. The general toxicity of TET with or without radiation was monitored via body weight changes during the experimental period, as shown in Fig. 6A.
No significant body weight changes among the six groups were found. Additionally, no other adverse effects, such as skin changes or hair loss, were observed in the mice post-radiation or with combination therapy. The survival curves in vivo were calculated using the Kaplan-Meier method, as presented in Fig. 6B. Mice were considered expired when the tumor volume reached $2,500 \mathrm{~mm}^{3}$.

\section{Discussion}

The antiproliferation effect of TET has been revealed in several different cell lines, and this inhibitory effect is reportedly exerted through apoptotic pathways (27-30). Ionizing radiation may cause DNA damage and induce apoptosis through the intrinsic pathway. Ionizing radiation may also cause rapid sphigomyelin hydrolysis to ceramide and initiate the extrinsic apoptotic pathway. In the present study, the $\mathrm{IC}_{50}$ of TET on CT26/tk-luc cells was $10 \mu \mathrm{M}$ (Fig. 1C). The survival curves of radiation alone and radiation combined with $20 \mu \mathrm{M}$ TET were similar and exhibited the same slope (Fig. 1D). This is indicative of an additive effect of combined therapeutic drug and ionizing radiation. This finding is different from that reported by Sun et al, who found a synergistic effect using a human CNE nasopharyngeal carcinoma cell line (30). This difference in radiosensitivity may be due to the following reasons: Firstly, the inherent radiosensitivity among various cancer cell lines is different; secondly, the TET dose used in combination with radiation was $20 \mu \mathrm{M}$ in the present study vs. $2 \mu \mathrm{M}$ in the study by Sun et al; thirdly, the sequence of the two treatments may affect the outcome. In this study, CT26/tk-luc cells were treated with $20 \mu \mathrm{M}$ TET for $3 \mathrm{~h}$ followed by various doses of radiation, while CNE cells were treated with $2 \mathrm{mM}$ TET post-irradiation in the study of Sun et al (30). The apoptosis was induced by TET in a dose-dependent manner, as demonstrated by the sub-G1 fraction and DNA fragmentation assays (Fig. 2A-C). In addition, cleaved caspase-3 in CT26/tk-luc cells treated with TET with or without radiation was also identified (Fig. 3A and B). The non-invasive BLI modality used for tumor growth monitoring obtains high-throughput and real-time images of therapeutic efficacy in animals (24). In the present study, the CT26/tk-luc cell line carrying HSV-1 thymidine kinase and firefly luciferase dual reporter genes was suitable for BLI and nuclear imaging, such as gamma scintigraphy (Fig. 5A and B).

Animals bearing CT26/tk-luc tumor xenografts treated with 5,10 or $20 \mathrm{mg} / \mathrm{kg}$ TET exhibited similar inhibition rates of tumor growth, since the total dosage was the same. The control of tumor growth was significantly improved in the radiation alone and combination groups compared with the control and TET alone groups. Notably, TET pretreatment followed by radiation exhibited a synergistic effect on tumor control, rather than an additive effect as determined in the in vitro study (Fig. 1D and Table I). The therapeutic ER for the combination group in vivo was 1.45. The enhancement in radiosensitization effect of TET in vivo may be partly due to the biochemical metabolism of TET into active ingredients. Alternatively, the enhanced effect may involve modulation of the tumor microenvironment, such as downregulation of the ERK/NF-kB or Akt/NF-kB/MMP-9 signaling pathways, which can be induced by ionizing radiation (31-33). Activation 
of these signaling pathways often results in an increase in effector proteins that are involved in tumor angiogenesis, anti-apoptosis, proliferation and invasion (31). However, this phenomenon requires further investigation. In addition, no general toxic effects were found in all experimental groups. These results demonstrated that TET is a radiosensitizer with a synergistic effect in vivo, and may be used in combination with ionizing radiation for cancer treatment in the clinic.

In conclusion, the cytotoxic effect of TET on CT26/tk-luc cells occured via the induction of apoptosis, and the combined effect of TET with ionizing radiation was additive in vitro. However, the combined effect of TET with ionizing radiation was synergistic in a CT26/tk-luc tumor-bearing animal model, and may have potential applications as a treatment adjuvant for cancer chemoradiotherapy.

\section{Acknowledgements}

The authors thank both the National Science Council (Taipei, Taiwan) and the Taipei City Hospital (Taipei, Taiwan) for the financial support.

\section{Funding}

The present study was supported by grants NSC942314-B-010-003 from the National Science Council (Taipei, Taiwan), and 95003-62-121 from the Taipei City Hospital Research Program (Taipei, Taiwan).

\section{Availability of data and materials}

The analyzed datasets generated during the study are available from the corresponding author on reasonable request.

\section{Authors' contributions}

WCL, WHW, YJC and JJH contributed to the conception and design of the study. WCL, WHW, YHL and SYC performed the experiments and wrote the first draft of the manuscript. JDL, YJC and JJH organized the research, contributed to the revision of the manuscript and gave final approval of the version to be published. All authors read and approved the manuscript and agree to be accountable for all aspects of the research in ensuring that the accuracy or integrity of any part of the work are appropriately investigated and resolved.

\section{Ethics approval and consent to participate}

The animal use protocol listed below (PDF file: 950501_ IACUC_Jeng-Jong Hwang.pdf) has been reviewed and approved by the Institutional Animal Care and Use Committee (IACUC).

\section{Patient consent for publication}

Not applicable.

\section{Competing interests}

The authors declare that they have no competing interests.

\section{References}

1. Siegel RL, Miller KD and Jemal A: Cancer statistics, 2015. CA Cancer J Clin 65: 5-29, 2015.

2. Sebag-Montefiore D, Stephens RJ, Steele R, Monson J, Grieve R, Khanna S, Quirke P, Couture J, de Metz C, Myint AS, et al: Preoperative radiotherapy versus selective postoperative chemoradiotherapy in patients with rectal cancer (MRC CR07 and NCIC-CTG C016): A multicentre, randomised trial. Lancet 373: 811-820, 2009.

3. Lin JK, Lee LK, Chen WS, Lin TC, Jiang JK, Yang SH, Wang HS, Chang SC, Lan YT, Lin CC, et al: Concurrent chemoradiotherapy followed by metastasectomy converts to survival benefitin stage IV rectum cancer. J Gastrointest Surg 16: 1888-1896, 2012.

4. Abbasakoor F, Vaizey CJ and Boulos PB: Improving the morbidity of anorectal injury from pelvic radiotherapy. Colorectal Dis 8: 2-10, 2006.

5. Chen YJ: Potential role of tetrandrine in cancer therapy. Acta Pharmacol Sin 23: 1102-1106, 2002.

6. Lai JH: Immunomodulatory effects and mechanisms of plant alkaloid tetrandrine in autoimmune diseases. Acta Pharmacol Sin 23: 1093-1101, 2002.

7. Wang H, Liu T, Li L, Wang Q, Yu C, Liu X and Li W: Tetrandrine is a potent autophagy agonist via activated intracellular reactive oxygen species. Cell Biosci 5: 4, 2015.

8. Liu T, Liu X and Li W: Tetrandrine, a Chinese plant-derived alkaloid, is a potential candidate for cancer chemotherapy. Oncotarget 7: 40800-40815, 2016

9. Wong VK, Zeng W, Chen J, Yao XJ, Leung EL, Wang QQ, Chiu P, Ko BCB and Law BYK: Tetrandrine, an activator of autophagy, induces autophagic cell death via PKC- $\alpha$ inhibitionand mTOR-dependent mechnisms. Front Pharmacol 8: 351, 2017.

10. Lai YL, Chen YJ, Wu TY, Wang SY, Chang KH, Chung CH and Chen ML: Induction of apoptosis in human leukemic U937 cells by tetrandrine. Anticancer Drugs 9: 77-81, 1998.

11. Lee JH, Kang GH, Kim KC, Kim KM, Park DI, Choi BT, Kang HS, Lee YT and Choi YH: Tetrandrine-induced cell cycle arrest and apoptosis in A549 human lung carcinoma cells. Int J Oncol 21, 1239-1244, 2002.

12. Yoo SM, Oh SH, Lee SJ, Lee BW, Ko WG, Moon CK and Lee BH: Inhibition of proliferation and induction of apoptosis by tetrandrine in HepG2 cells. J Ethnopharmacol 81: 225-229, 2002.

13. Chen Y, Chen JC and Tseng SH: Effects of tetrandrine plus radiation on neuroblastoma cells. Anticancer Res 29: 3163-3171, 2009.

14. Wu K, Zhou M, Wu QX, Yuan SX, Wang DX, Jin JL, Huang J, Yang JQ, Sun WJ, Wan LH and He BC: The role of IGFBP-5 in mediating the anti-proliferation effect of tetrandrine in human colon cancer cells. Int J Oncol 46: 1205-1213, 2015.

15. Chang KH, Chen ML, Chen HC, Huang YW, Wu TY and Chen YJ: Enhancement of radiosensitivity in human glioblastoma U138MG cells by tetrandrine. Neoplasma 46: 196-200, 1999.

16. Gupta S: Molecular steps of death receptor and mitochondrial pathways of apoptosis. Life Sci 69: 2957-2964, 2001.

17. Salvesen GS: Caspase 8: Igniting the death machine. Structure 7: R225-R229, 1999.

18. Stennicke HR, Jürgensmeier JM, Shin H, Deveraux Q, Wolf BB Yang X, Zhou Q, Ellerby HM, Ellerby LM, Bredesen D, et al: Pro-caspase-3 is a major physiologic target of caspase-8. J Biol Chem 273: 27084-27090, 1998.

19. Haimovitz-Friedman A, Kan CC, Ehleiter D, Persaud RS, McLoughlin M, Fuks Z and Kolesnick RN: Ionizing radiation acts on cellular membranes to generate ceramide and initiate apoptosis. J Exp Med 180: 525-535, 1994.

20. Yu J, Liu F, Sun M, Sun Z and Sun S: Enhancement of radiosensitivity and the potential mechanism on human esophageal carcinoma cells by tetrandrine. Cancer Biother Radiopharm 26: 437-442, 2011

21. Chang YF, Lin YY, Wang HE, Liu RS, Pang F and Hwang JJ: Monitoring of tumor growth and metastasis potential in MDA-MB-435s/tk-luc human breast cancer xenografts. Nucl Instrum Meth A 571: 155-159, 2007.

22. Chen CC, Hwang JJ, Ting G, Tseng YL, Wang SJ and Peng JW: Monitoring and quantitative assessment of tumor burden using in vivo bioluminescence imaging. Nucl Instrum Meth A 571: 437-441, 2007.

23. Jänicke RU, Sprengart ML, Wati MR and Porter AG: Caspase-3 is required for DNA fragmentation and morphological changes associated with apoptosis. J Biol Chem 273: 9357-9360, 1998. 
24. Burgos JS, Rosol M, Moats RA, Khankaldyyan V, Kohn DB, Nelson MD Jr and Laug WE: Time course of bioluminescent signal in orthotopic and heterotopic brain tumors in nude mice. Biotechniques 34: 1184-1188, 2003.

25. Cividalli A, Ceciarelli F, Livdi E, Altavista P, Cruciani G, Marchetti P and Danesi DT: Radiosensitization by oxaliplatin in a mouse adenocarcinoma: Influence of treatment schedule. Int J Radiat Oncol Biol Phys 52: 1092-1098, 2002.

26. Heckelsmiller K, Rall K, Beck S, Schlamp A, Seiderer J, Jahrsdörfer B, Krug A, Rothenfusser S, Endres S and Hartmann G: Peritumoral CpG DNA elicits a coordinated response of CD8 T cells and innate effectors to cure established tumors in a murine colon carcinoma model. J Immunol 169: 3892-3899, 2002.

27. Jang BC, Lim KJ, Paik JH, Cho JW, Baek WK, Suh MH, Park JB, Kwon TK, Park JW, Kim SP, et al: Tetrandrine-induced apoptosis is mediated by activation of caspases and PKC- $\delta$ in U937 cells. Biochem Pharmacol 67: 1819-1829, 2004.

28. Kuo PL and Lin CC: Tetrandrine-induced cell cycle arrest and apoptosis in Hep G2 cells. Life Sci 73: 243252, 2003.

29. Ng LT, Chiang LC, Lin YT and Lin CC: Antiproliferative and apoptotic effects of tetrandrine on different human hepatoma cell lines. Am J Chin Med 34: 125-135, 2006.
30. Sun X, Xu R, Deng Y, Cheng H, Ma J, Ji J and Zhou Y: Effects of Tetrandrine on apoptosis and radiosensitivity of nasopharyngeal carcinoma cell line CNE. Acta Biochim Biophys Sin 39: 869-878, 2007.

31. Hsu FT, Chang B, Chen JC, Chiang IT, Liu YC, Kwang WK and Hwang JJ: Synergistic effect of sorafenib and radiation on human oral carcinoma in vivo. Sci Rep 5: 15391, 2015.

32. Wu CJ, Wang YH, Lin CJ, Chen $\mathrm{HH}$ and Chen YJ: Tetrandrine down-regulaties ERK/NF- $\mathrm{BB}$ signaling and inhibits activtion of mesangial cells. Toxicol in vitro 25: 1834-1840, 2011.

33. Chen S, Liu W, Wang K, Fan Y, Chen J, Ma J, Wang X, He D, Zeng $\mathrm{J}$ and $\mathrm{Li} \mathrm{L}$ : Tetrandrine inhibits migration and invasion of human renal cell carcinoma by regulating Akt/NF-кB/MMP-9 signaling. PLoS One 12: e0173725, 2017.

(i) $\Theta$ This work is licensed under a Creative Commons Attribution-NonCommercial-NoDerivatives 4.0 International (CC BY-NC-ND 4.0) License. 